\title{
BOUNDARY VALUE PROBLEMS FOR THE DIFFUSION EQUATION WITH PIECEWISE CONTINUOUS TIME DELAY
}

\section{JOSEPH WIENER}

Department of Mathematics

University of Texas - Pan American

Edinburg, Texas 78539, U.S.A

\section{LOKENATH DEBNATH}

Department of Mathematics

University of Central Florida

Orlando, Florida 32816, U S A

(Received November 30, 1995 and in revised form January 20, 1996)

\begin{abstract}
A study is made of partial differential equations with piecewise constant arguments. Boundary value problems for three types of equations are discussed delayed; alternately of advanced and retarded type; and most importantly, an equation of neutral type (that is, including the derivative at different values of time $t$ ).
\end{abstract}

KEY WORDS AND PHRASES: Partial differential equations, piecewise constant arguments, oscillations, and stability.

1991 AMS SUBJECT CLASSIFICATION CODES: 35A05, 35B25, 35L10, 34K25.

\section{INTRODUCTION}

Functional differential equations (FDE) with delay provide a mathematical model for a physical or biological system in which the rate of change of the system depends upon its past history. The theory of FDE with continuous arguments is well developed and has numerous applications in natural and engineering sciences. This paper continues our earlier work in an attempt to extend this theory to differential equations with discontinuous argument deviations. In articles [1-5], ordinary differential equations with arguments having intervals of constancy have been studied. Such equations represent a hybrid of continuous and discrete dynamical systems and combine properties of both differential and difference equations. They include as particular cases loaded and impulsive equations, hence their importance in control theory and in certain biomedical models Continuity of a solution at a point joining any two consecutive intervals implies recursion relations for the values of the solution at such points. Therefore, differential equations with piecewise continuous argument (EPCA) are intrinsically closer to difference rather than to differential equations. In [6] boundary value problems for some linear EPCA in partial derivatives were considered and the behavior of their solutions studied. The results were also extended to equations with positive definite operators in Hilbert spaces. In [7] initial value problems were studied for EPCA in partial derivatives. A class of loaded equations that arise in solving certain inverse problems was explored within the general framework of differential equations with piecewise continuous delay The purpose of the present note is to investigate the asymptotic behavior of the solutions, especially their oscillatory properties, of a boundary value problem for some EPCA of parabolic type. For a rather comprehensive addition to the growing body of literature on EPCA the reader is referred to [8]. 


\section{A COMPARISON OF TWO EPCA}

The equation

$$
u_{t}(x, t)=a^{2} u_{x x}(x, t)-b u(x, t)
$$

describes heat flow in a rod with both diffusion $a^{2} u_{x x}$ along the rod and heat loss (or gain) across the lateral sides of the rod. Measuring the lateral heat change at discrete moments of time leads to the equation with piecewise continuous delay

$$
u_{t}(x, t)=a^{2} u_{x x}(x, t)-b u(x,[t])
$$

which was investigated in [6]. Here $[\cdot]$ designates the greatest integer function and $(x, t) \in[0,1] \times[0, \infty)$ The problem posed in [6] for Eq. (2.2) consists of the boundary conditions

$$
u(0, t)=0, \quad u(1, t)=0
$$

and the initial condition

$$
u(x, 0)=u_{0}(x)
$$

and the solution is sought in the form

$$
u(x, t)=X(x) T(t) .
$$

Then separation of variables leads to the BVP

$$
X^{\prime}+\lambda^{2} X=0, \quad X(0)=X(1)=0,
$$

with the orthonormal set of solutions

$$
X_{\jmath}(x)=\sqrt{2} \sin (\pi j x)
$$

on $[0,1]$, and to the equation

$$
T_{\jmath}^{\prime}(t)=-a^{2} \pi^{2} j^{2} T_{\jmath}(t)-b T_{\jmath}([t]) .
$$

Let $T_{n j}(t)$ denote a solution of (2.8) on the interval $n \leq t<n+1$, where $n$ is a nonnegative integer Then

$$
T_{n j}^{\prime}(t)=-a^{2} \pi^{2} j^{2} T_{n j}(t)-b T_{n j}(n),
$$

and the general solution of this equation is

$$
T_{n j}(t)=C_{n j} e^{-a^{2} \pi^{2} j^{2}(t-n)}-\frac{b}{a^{2} \pi^{2} j^{2}} T_{n j}(n) .
$$

We put here $t=n$ and get

$$
C_{n \jmath}=\left(1+\frac{b}{a^{2} \pi^{2} \jmath^{2}}\right) T_{n \jmath}(n)
$$

that is,

$$
T_{n \jmath}(t)=E_{\jmath}(t-n) T_{n \jmath}(n),
$$

where

$$
E_{j}(t)=e^{-a^{2} \pi^{2} j^{2} t}-\frac{b}{a^{2} \pi^{2} j^{2}}\left(1-e^{-a^{2} \pi^{2} j^{2} t}\right) .
$$

At $t=n+1$ we have

$$
T_{n \jmath}(n+1)=E_{\jmath}(1) T_{n \jmath}(n)
$$


and since

$$
T_{n j}(n+1)=T_{n+1, j}(n+1),
$$

then

$$
T_{n+1, \jmath}(n+1)=E_{\jmath}(1) T_{n \jmath}(n)
$$

and

$$
T_{n \jmath}(n)=E_{n}^{n}(1) T_{0 \jmath}(0) .
$$

Therefore,

$$
T_{n \jmath}(t)=E_{\jmath}(t-n) E_{\jmath}^{n}(1) T_{0 \jmath}(0)
$$

and

$$
u_{n}(x, t)=\sum_{\jmath=1}^{\infty} \sqrt{2} E_{\jmath}^{n}(1) T_{0 \jmath}(0) E_{\jmath}(t-n) \sin (\pi j x),
$$

where $u_{n}(x, t)$ designates the solution of BVP (2.2), (2.3), (2.4) in $[0,1] \times[n, n+1]$. Putting $t=0$, $n=0$ gives

$$
u_{0}(x)=\sum_{j=1}^{\infty} T_{0 j}(0) \sqrt{2} \sin (\pi j x)
$$

and

$$
T_{0 j}(0)=\sqrt{2} \int_{0}^{1} u_{0}(x) \sin (\pi j x) d x .
$$

Along with Eq. (2.2), we study the equation

$$
u_{t}(x, t)=a^{2} u_{x x}(x, t)-b u\left(x,\left[t+\frac{1}{2}\right]\right)
$$

under conditions (2.3), (2 4)

DEFINITION. A function $u(x, t)$ is said to be a solution of the above BVP if it satisfies the conditions (i) $u(x, t)$ is continuous in $G=[0,1] \times[0, \infty)$; (ii) $u_{t}$ and $u_{x x}$ exist and are continuous in $G$, with the possible exception of the points $\left(x, n+\frac{1}{2}\right)$, where one-sided derivatives exist $(n=0,1,2, \ldots)$; (ii) $u(x, t)$ satisfies Eq. $(2.16)$ in $G$, with the possible exception of the points $\left(x, n+\frac{1}{2}\right)$, and conditions (2.3), (2.4).

Again, the solution is sought in form (2.5), and separation of variables generates the eigenfunctions (2.7) and leads to the EPCA

$$
T_{\jmath}^{\prime}(t)=-a^{2} \pi^{2} j^{2} T_{\jmath}(t)-b T_{\jmath}\left(\left[t+\frac{1}{2}\right]\right)
$$

Eq. (2.17) is of considerable interest, since the argument deviation

$$
\tau(t)=t-\left[t+\frac{1}{2}\right]
$$

changes the sign in each interval $\left(n-\frac{1}{2}, n+\frac{1}{2}\right)$, with integer $n$. Indeed, $\tau(t)<0$ for $n-\frac{1}{2} \leq t<n$ and $\tau(t)>0$ for $n<t<n+\frac{1}{2}$, which means that Eq. (2.17) is alternately of advanced and retarded type. Assume that $T_{n j}(t)$ is a solution of (2.17) on the interval $\left[n-\frac{1}{2}, n+\frac{1}{2}\right]$. Then Eq. (2.17) changes to (29), with the solution (2.11). At $t=n+\frac{1}{2}$ we have

$$
T_{n j}\left(n+\frac{1}{2}\right)=E_{j}\left(\frac{1}{2}\right) T_{n j}(n)
$$


and since

$$
T_{n+1, \jmath}(t)=E_{\jmath}(t-n-1) T_{n+1, \jmath}(n+1),
$$

then

$$
T_{n+1, \jmath}\left(n+\frac{1}{2}\right)=E_{\jmath}\left(-\frac{1}{2}\right) T_{n+1, \jmath}(n+1) .
$$

Furthermore, continuity of the solution $T_{\jmath}(t)$ implies

$$
T_{n+1, J}\left(n+\frac{1}{2}\right)=T_{n \jmath}\left(n+\frac{1}{2}\right)
$$

and therefore

$$
E_{\jmath}\left(-\frac{1}{2}\right) T_{n+1, \jmath}(n+1)=E_{\jmath}\left(\frac{1}{2}\right) T_{n \jmath}(n),
$$

whence

$$
T_{n+1, \jmath}(n+1)=\frac{E_{\jmath}(1 / 2)}{E_{\jmath}(-1 / 2)} T_{n \jmath}(n)
$$

From here,

$$
T_{n j}(n)=E_{\jmath}^{n}\left(\frac{1}{2}\right) E_{\jmath}^{-n}\left(-\frac{1}{2}\right) T_{0 \jmath}(0)
$$

and

$$
T_{n \jmath}(t)=E_{\jmath}(t-n) E_{\jmath}^{n}\left(\frac{1}{2}\right) E_{\jmath}^{-n}\left(-\frac{1}{2}\right) T_{0 \jmath}(0) .
$$

The solution $u_{n}(x, t)$ of BVP (2.17), (2.3), (2.4) in the region $[0,1] \times\left[n-\frac{1}{2}, n+\frac{1}{2}\right]$ is given by the formula

$$
u_{n}(x, t)=\sum_{\jmath=1}^{\infty} \sqrt{2} E_{\jmath}^{n}\left(\frac{1}{2}\right) E_{\jmath}^{-n}\left(-\frac{1}{2}\right) T_{0 \jmath}(0) E_{\jmath}(t-n) \sin (\pi j x),
$$

where $E_{\jmath}(t)$ is defined by (2.12).

THEOREM 2.1. For

$$
-a^{2} \pi^{2}<b<a^{2} \pi^{2}\left(e^{a^{2} \pi^{2}}+1\right) /\left(e^{a^{2} \pi^{2}}-1\right)
$$

the solution (2.15) of Eq. (2.2) tends to zero as $t \rightarrow \infty$, uniformly with respect to $x$

PROOF. From (2.15) it follows that the assertion is true if $\left|E_{\jmath}(1)\right|<1$. Solving the inequalities

$$
-1<e^{-a^{2} \pi^{2} j^{2}}-\frac{b}{a^{2} \pi^{2} j^{2}}\left(1-e^{-a^{2} \pi^{2} j^{2}}\right)<1
$$

for $b$ proves the proposition. Furthermore, $u(x, t)$ approaches zero with an exponential rate

THEOREM 2.2. For

$$
b>-a^{2} \pi^{2}
$$

the solution (2.20) of Eq. (2.16) tends to zero as $t \rightarrow \infty$, uniformly with respect to $x$.

PROOF. From (2.20) we see that the assertion is valid if $\left|E_{j}(1 / 2) E_{\jmath}^{-1}(-1 / 2)\right|<1$, where $E_{\jmath}(t)$ is given by (2.12). Considering the case $E_{\jmath}(-1 / 2)>0$ gives

$$
b>-2 \mu, e^{\mu,} /\left(e^{\mu,}-1\right)
$$


with the notation

$$
\mu_{\jmath}=a^{2} \pi^{2} j^{2} / 2 .
$$

From $E_{\jmath}(1 / 2)<E_{\jmath}(-1 / 2)$ we obtain the inequality

$$
b>-2 \mu_{\jmath}
$$

which is stronger than (2 23), and so (2 23) should be omitted Furthermore, $E_{\jmath}(1 / 2)>-E_{\jmath}(-1 / 2)$ implies that

$$
b>-2 \mu_{\jmath}\left(e^{\mu_{\jmath}}+e^{-\mu_{\jmath}}\right) /\left(e^{\mu_{\jmath}}+e^{-\mu_{\jmath}}-2\right),
$$

and since this inequality is weaker than (2.25) it may be disregarded On the other hand, the case $E_{\jmath}(-1 / 2)<0$ is equivalent to

$$
b<-2 \mu_{j} e^{\mu_{j}} /\left(e^{\mu,}-1\right),
$$

and the inequality $E_{\jmath}(1 / 2)>E_{\jmath}(-1 / 2)$ leads to

$$
b<-2 \mu_{\jmath} .
$$

The latter inequality may be ignored because it is weaker than (2.26) From $E_{\jmath}(1 / 2)<-E_{\jmath}(-1 / 2)$ it follows that

$$
b<-2 \mu_{\jmath}\left(e^{\mu_{\jmath}}+e^{-\mu_{\jmath}}\right) /\left(e^{\mu_{\jmath}}+e^{-\mu_{\jmath}}-2\right) .
$$

The latter inequality should be omitted since (2.26) is more stringent. On the other hand, (2.26) cannot hold true for all values of $j$, and we must retain only (2.25). This inequality is valid for all $j$ if (2.22) takes place. Comparing inequalities (2.21) and (2.22) shows that the stability interval for Eq. (2.17) is larger than the stability interval for Eq. (2.8).

THEOREM 2.3. Each solution of Eq. (2.8) has a zero in the interval $[n, n+1]$ if

$$
b>a^{2} \pi^{2} /\left(e^{a^{2} \pi^{2}}-1\right) .
$$

The same is true for $\mathrm{Eq}(2.17)$ if

$$
b>(a \pi)^{2} /\left(e^{a^{2} \pi^{2} / 2}-1\right) .
$$

PROOF. From Eq. (2.13) we have

$$
T_{n \jmath}(n+1)=E_{\jmath}^{n+1}(1) T_{0 \jmath}(0)
$$

and

$$
T_{n \jmath}(n) T_{n \jmath}(n+1)=E_{\jmath}^{2 n+1}(1) T_{0 \jmath}^{2}(0) .
$$

Hence,

$$
T_{n \jmath}(n) T_{n \jmath}(n+1)<0 \quad \text { if } \quad E_{\jmath}(1)<0 .
$$

The latter inequality is equivalent to

$$
b>a^{2} \pi^{2} j^{2} /\left(e^{a^{2} \pi^{2} j^{2}}-1\right),
$$

which holds true for all $\jmath$ under condition (2 27). On the other hand, $(2.28)$ is derived by examining the inequality

$$
E_{\jmath}(1 / 2) E_{\jmath}(-1 / 2)<0
$$


Inequalities (2.27) and (2 28) represent sufficient conditions of oscillation for Eqs (2 8) and (2 17), for all values of $j$ simultaneously

THEOREM 2.4. Each solution of Eq. (2.8) is nonoscillatory, for all $j$, if $b<0$ For any $b>0$ and sufficiently large $j$, the functions $T_{n_{j}}(t)$ are oscillatory.

PROOF. The inequality $E_{\jmath}(1)>0$ is equivalent to

$$
b<a^{2} \pi^{2} j^{2} /\left(e^{a^{2} \pi^{2} j^{2}}-1\right)
$$

and holds true for all $j$ only if $b<0$ Since its right-hand side tends to zero as $j \rightarrow \infty$, the above inequality breaks down for $b>0$ and all sufficiently large $j$. Therefore, in this case the solutions $T_{n \jmath}(t)$ of (2 8) oscillate, in sharp contrast to the functions $T_{\jmath}(t)$ in the Fourier expansion for the solution of the equation $u_{t}=a^{2} u_{x x}-b u$ without time delay.

THEOREM 2.5. Each solution of Eq. (2.17) is nonoscillatory, for all $j$, if

$$
-a^{2} \pi^{2} /\left(e^{a^{2} \pi^{2} / 2}-1\right)<b<0 .
$$

For any $b>0$ and sufficiently large $j$, the functions $T_{n j}(t)$ are oscillatory Furthermore, if

$$
b<-a^{2} \pi^{2} m^{2} /\left(e^{a^{2} \pi^{2} m^{2} / 2}-1\right)
$$

then the functions $T_{n 1}(t), \ldots, T_{n m}(t)$ oscillate.

PROOF. From the inequalities $E_{\jmath}(-1 / 2)>0$ and $E_{\jmath}(1 / 2)>0$ it follows that

$$
-2 \mu_{\jmath} e^{\mu_{\jmath}} /\left(e^{\mu_{\jmath}}-1\right)<b<2 \mu_{\jmath} /\left(e^{\mu_{\jmath}}-1\right),
$$

where $\mu_{j}$ is given by (2.24). The left of these inequalities holds true for all $j$ if

$$
b>-a^{2} \pi^{2} /\left(e^{a^{2} \pi^{2} / 2}-1\right),
$$

and the right one is satisfied for all $j$ if $b<0$. The inequalities $E_{\jmath}(-1 / 2)<0$ and $E_{\jmath}(1 / 2)<0$ are contradictory. On the other hand, the right side of the inequality

$$
b<2 \mu_{\jmath} /\left(e^{\mu,}-1\right)
$$

tends to zero as $j \rightarrow \infty$, and therefore the inequality breaks down for any given $b>0$ and all sufficiently large $j$. Moreover, the left side of the inequality

$$
-2 \mu_{j} e^{\mu,} /\left(e^{\mu,}-1\right)<b
$$

monotonically decreases to $-\infty$ as $j \rightarrow \infty$. Hence, if this inequality fails for $j=m$, the same is true also for $j<m$.

THEOREM 2.6. If, for some integer $m \geq 1$,

$$
b=a^{2} \pi^{2} m^{2}\left(e^{a^{2} \pi^{2} m^{2}}+1\right) /\left(e^{a^{2} \pi^{2} m^{2}}-1\right)
$$

then the solution $T_{m}(t)$ of Eq. (2.8) is a periodic function with period 2. The same is true for the solution of $\mathrm{Eq}$ (2.17) if

$$
b=2 \mu_{m}\left(e^{\mu_{m}}+e^{-\mu_{m}}\right) /\left(2-e^{\mu_{m}}-e^{-\mu_{m}}\right)
$$

where $\mu_{m}$ is given in (2.24).

PROOF. Since the initial value problem for Eq. (2.8) has a unique solution on each unit interval with integral endpoints, we have only to show that

$$
T_{m}(n+2)=T_{m}(n), \quad n=0,1,2, \ldots .
$$

Consider solution (2.14) for $j=m$ on the intervals $[n, n+1]$ and $[n+2, n+3]$, that is, 


$$
T_{m}(t)=E_{m}(t-n) E_{m}^{n}(1) T_{0 m}(0), \quad n \leq t \leq n+1
$$

and

$$
T_{m}(t)=E_{m}(t-n-2) E_{m}^{n+2}(1) T_{0 m}(0), \quad n+2 \leq t \leq n+3 .
$$

At $t=n$ and $t=n+2$ these formulas respectively yield

$$
T_{m}(n)=E_{m}^{n}(1) T_{0 m}(0) \text { and } T_{m}(n+2)=E_{m}^{n+2}(1) T_{0 m}(0) .
$$

Since hypothesis $(230)$ implies $E_{m}(1)=1$, then $T_{m}(n+2)=T_{m}(n)$, which proves the theorem for $\mathrm{Eq}$ (2.8) The proof for $\mathrm{Eq}(217)$ is analogous Condition (2.31) is equivalent to $E_{m}(1 / 2)=-E_{m}(-1 / 2)$ It remains to note that the value of $b$ in $(2.30)$ is positive, and the value of $b$ in (2.31) is negative.

\section{A PARABOLIC EPCA OF NEUTRAL TYPE}

Consider the boundary value problem (BVP) consisting of the equation

$$
u_{t}(x, t)=a^{2} u_{x x}(x, t)+b u_{t}(x,[t])
$$

and conditions (2.3) and (2.4). This equation is of neutral type since it includes the derivative $u_{t}$ at different values of $t$.

Let $u_{n}(x, t)$ be the solution of the given problem on the interval $n \leq t \leq n+1$. Then $u_{n}(x, t)$ satisfies the equation

$$
\frac{\partial u_{n}(x, t)}{\partial t}=a^{2} \frac{\partial^{2} u_{n}(x, t)}{\partial x^{2}}+b \frac{\partial^{2} u_{n}}{\partial x^{2}}\left(x, n^{+}\right)
$$

with boundary conditions (2.3) and the initial condition

$$
u_{n}\left(x, n^{+}\right)=u_{n}(x)
$$

where $u_{n}(x)$ is yet unknown. The solution of (3.2) is sought in form (2.5), and separation of variables generates BVP (2.6) with eigenfunctions (2.7) and the ODE

$$
T_{n \jmath}^{\prime}=-a^{2} \lambda_{\jmath}^{2} T_{n \jmath}(t)+b T_{n \jmath}^{\prime}\left(n^{+}\right), \quad \lambda_{\jmath}=\pi j .
$$

Then the series

$$
u_{n}(x, t)=\sum_{\jmath=1}^{\infty} X_{\jmath}(x) T_{n \jmath}(t)
$$

represents a formal solution of problem (3.2), (2.3). Turning to Eq. (3.3), its general solution on the interval $n \leq t<n+1$ is

$$
T_{n \jmath}(t)=C_{n \jmath} e^{-a^{2} \lambda_{j}^{2}(t-n)}+\frac{b}{a^{2} \lambda_{\jmath}^{2}} T_{n \jmath}^{\prime}\left(n^{+}\right) .
$$

At $t=n^{+}, \mathrm{Eq}(3.3)$ gives

$$
T_{n j}^{\prime}\left(n^{+}\right)=-a^{2} \lambda_{\jmath}^{2} T_{n j}(n)+b T_{n j}^{\prime}\left(n^{+}\right)
$$

whence

$$
T_{n \jmath}^{\prime}\left(n^{+}\right)=\frac{a^{2} \lambda_{\jmath}^{2}}{b-1} T_{n \jmath}(n), \quad b \neq 1 .
$$

Therefore,

$$
T_{n \jmath}(t)=C_{n \jmath} e^{a^{2} \lambda_{j}^{2}(t-n)}+\frac{b}{b-1} T_{n \jmath}(n) .
$$


At $t=n$, we have

$$
T_{n \jmath}(n)=C_{n \jmath}+\frac{b}{b-1} T_{n \jmath}(n),
$$

that is,

$$
C_{n \jmath}=\frac{1}{1-b} T_{n \jmath}(n)
$$

and

$$
T_{n \jmath}(t)=\frac{1}{b-1}\left(b-e^{-a^{2} \lambda_{\jmath}^{2}(t-n)}\right) T_{n_{\jmath}}(n) .
$$

Denote

$$
F_{\jmath}(t)=\frac{b-e^{-a^{2} \lambda_{j}^{2} t}}{b-1}
$$

At $t=n+1$, we have

$$
T_{n \jmath}(n+1)=F_{\jmath}(1) T_{n \jmath}(n)
$$

and since, by hypothesis (i) of the above definition,

$$
T_{n \jmath}(n+1)=T_{n+1, j}(n+1),
$$

then

$$
T_{n+1, \jmath}(n+1)=F_{j}(1) T_{n j}(n)
$$

and

$$
T_{n \jmath}(n)=F_{\jmath}^{n}(1) T_{0 j}(0) .
$$

Consequently,

$$
T_{n j}(t)=F_{j}(t-n) F_{\jmath}^{n}(1) T_{0 j}(0)
$$

and

$$
u_{n}(x, t)=\sum_{j=1}^{\infty} \sqrt{2} F_{\jmath}^{n}(1) T_{0 j}(0) F_{\jmath}(t-n) \sin (\pi j x) .
$$

The following theorems illustrate the far more complicated solution structure of Eq. (3.1) comparing with the diffusion equation without time delay.

THEOREM 3.1. For $b>1$, each function $T_{n j}(t)$ is monotone unbounded as $t \rightarrow+\infty$.

PROOF. If $b>1$, then (3.5) implies that $F_{\jmath}(t)>1$, for all $t>0$. Hence, $F_{\jmath}^{n}(1)$ grows exponentially as $t \rightarrow+\infty$, and the proof follows from (3.7)

THEOREM 3.2. For

$$
\frac{1}{2}\left(1+e^{-a^{2} \lambda_{1}^{2}}\right)<b<1
$$

each function $T_{n \jmath}(t)$ is unbounded and oscillating.

PROOF. From inequalities (3.9) it follows that $F_{\jmath}(1)<-1$, and therefore $F_{\jmath}^{[t]}(1)$ is unbounded and has a zero in each unit interval with integer endpoints.

THEOREM 3.3. For $b<0$, each function $T_{n j}(t)$ monotonically tends to zero as $t \rightarrow+\infty$

PROOF. The inequalities

$$
0<F_{\jmath}(1)<1
$$

imply that solution (3.7) is asymptotically stable and nonoscillatory. From (3 10) it follows that 


$$
b<e^{-a^{2} \lambda^{3}}
$$

Clearly the right-hand side of (3 11) vanishes as $j \rightarrow \infty$, and therefore the condition $b<0$ is necessary and sufficient for all $T_{n_{j}}(t)$ to approach zero monotonically.

THEOREM 3.4. If

$$
a^{2} \lambda_{1}^{2}>\ln 2 \text { and } e^{-a^{2} \lambda_{1}^{2}}<b<\frac{1}{2}
$$

then every solution (3 7) tends to zero and oscillates as $t \rightarrow+\infty$

PROOF. From the inequalities

$$
-1<F_{3}(1)<0,
$$

which imply asymptotic stability and oscillatory behavior of solution ( 37 ), it follows that

$$
e^{-a^{2} \lambda_{j}^{2}}<b<\frac{1}{2}\left(1+e^{-a^{2} \lambda_{j}^{2}}\right)
$$

These inequalities hold true for all $j$ simultaneously with (3.12)

THEOREM 3.5. For any coefficient $b$ that satisfies the inequalities

$$
\frac{1}{2}<b<1
$$

there exist infinitely many solutions $T_{n j}(t)$ which are unbounded and oscillating.

PROOF. For $b<1$, the inequality $F_{\jmath}(1)<-1$ which implies unboundedness and oscillation of $T_{n j}(t)$, is equivalent to

$$
b>\frac{1}{2}+\frac{1}{2} e^{-a^{2} \lambda^{2}}
$$

Clearly the right-hand side approaches $1 / 2$ as $j \rightarrow \infty$.

THEOREM 3.6. If $b<e^{-a^{2} \lambda_{m}^{2}}$, then the solutions $T_{1}(t), \ldots, T_{m}(t)$ of Eq. (3.3) monotonically tend to zero as $t \rightarrow+\infty$.

ACKNOWLEDGEMENT. This research was partially supported by NASA Grant NAG9-553

\section{REFERENCES}

[1] AFTABIZADEH, A.R. and WIENER, J., Oscillatory properties of first order linear functional differential equations, Applicable Anal. 20 (1985), 165-187.

[2] AFTABIZADEH, A.R., WIENER, J. and XU, J.-M., Oscillatory and periodic solutions of delay differential equations with piecewise constant argument, Proc. Amer. Math. Soc. 99 (1987), 673679 .

[3] COOKE, K.L. and WIENER, J., Retarded differential equations with piecewise constant delays, $J$. Math. Anal. App. 99 (1984), 265-297.

[4] COOKE, K.L. and WIENER, J., An equation alternately of retarded and advanced type, Proc. Amer. Math. Soc. 99 (1987), 726-732.

[5] WIENER, J. and COOKE, K.L., Oscillations in systems of differential equations with piecewise constant argument, J. Math. Anal. Appl. 137 (1989), 221-239.

[6] WIENER, J., Boundary-value problems for partial differential equations with piecewise constant delay, Internat. J. Math. and Math. Sci. 14 (1991), 301-321

[7] WIENER, J. and DEBNATH, L., Partial differential equations with piecewise constant delay, Internat. J. Math. and Math. Scl. 14 (1991), 485-496.

[8] WIENER, J., Generalized Solutions of Functional Differential Equations, World Scientific, Singapore, 1993 


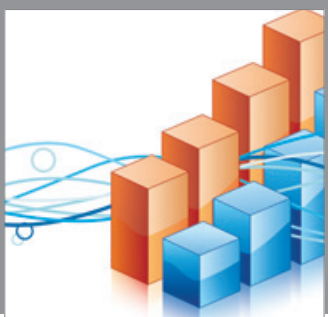

Advances in

Operations Research

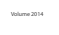

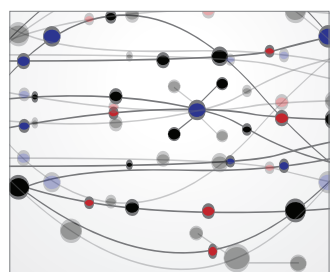

\section{The Scientific} World Journal
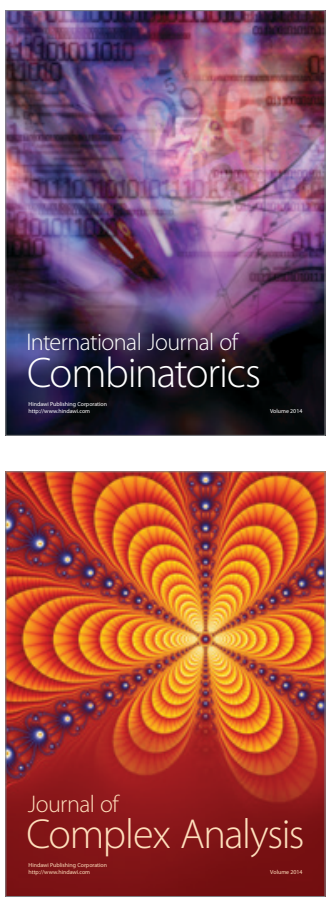

International Journal of

Mathematics and

Mathematical

Sciences
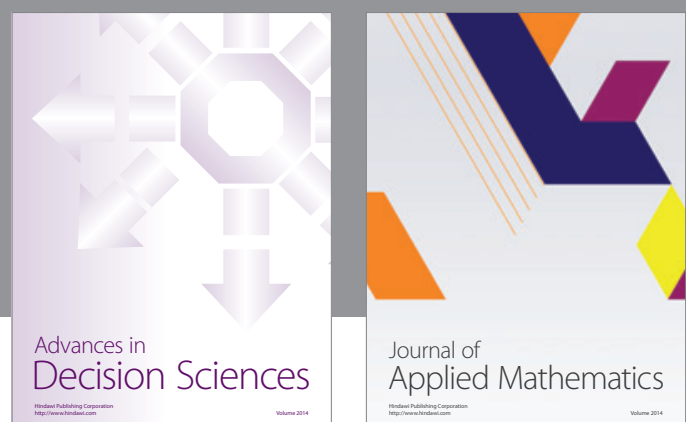

Journal of

Applied Mathematics
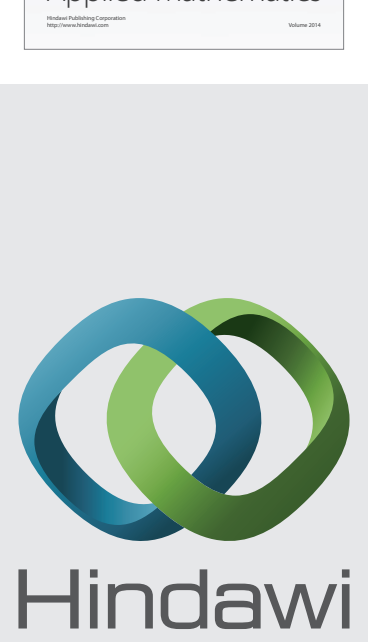

Submit your manuscripts at http://www.hindawi.com
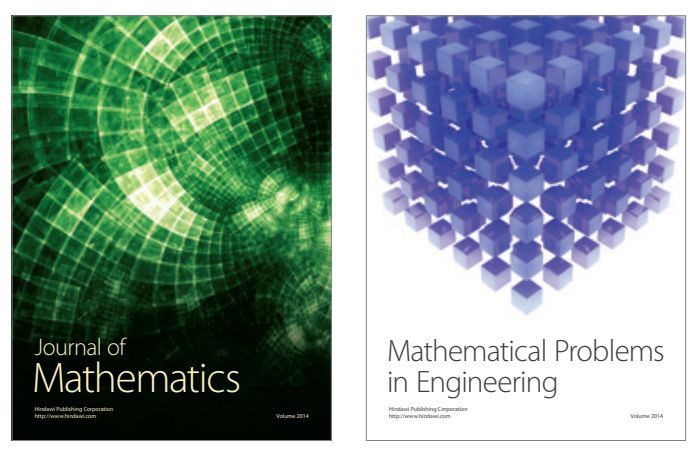

Mathematical Problems in Engineering
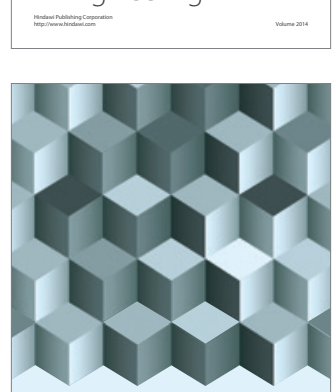

Journal of

Function Spaces
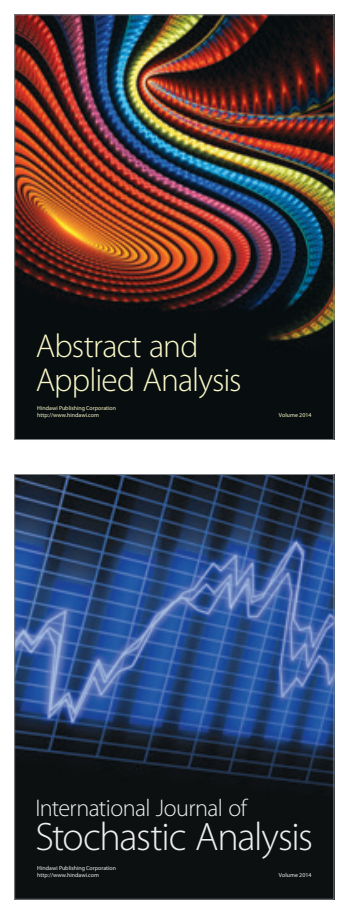

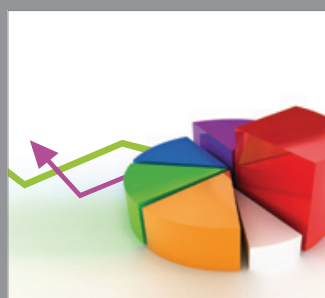

ournal of

Probability and Statistics

Promensencen
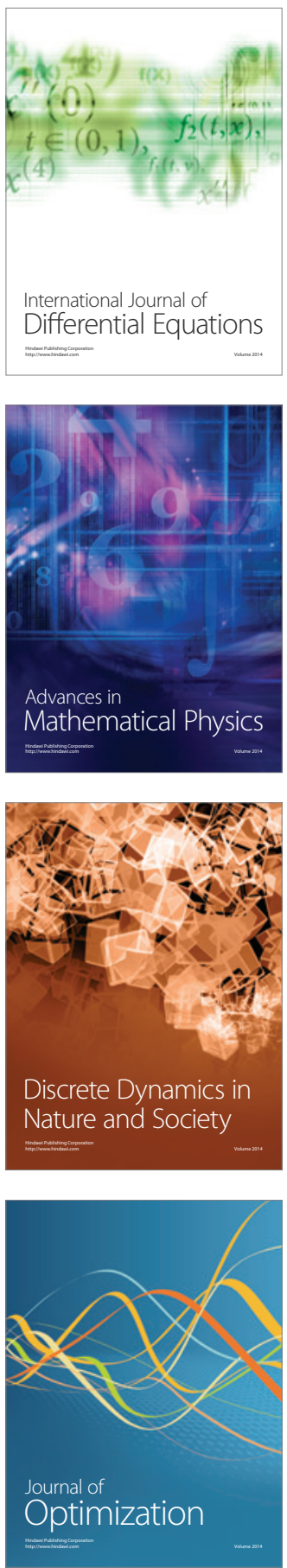